\title{
Observation of the Saturation of Langmuir Waves Driven by Ponderomotive Force in a Large Scale Plasma
}

\author{
R. K Kirkwood, D. S. Montgomery, B. B. Afeyan, J. D. Moody, \\ B. J. MacGowan, S. H. Glenzer, W. L. Kruer, K. G. Estabrook, \\ K. B. Wharton, E. A. Williams, R. L. Berger
}

June 22, 1997

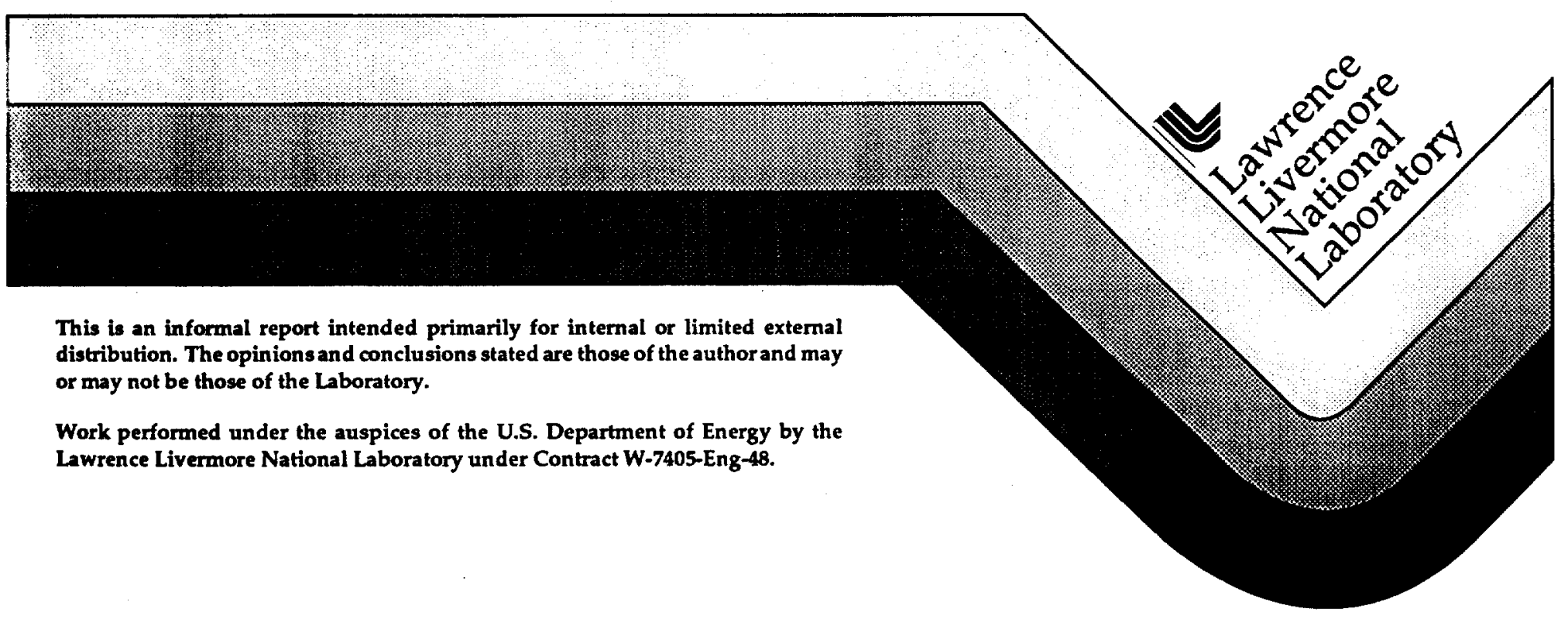




\section{DISCLAIMER}

This document was prepared as an account of work sponsored by an agency of the United States Government. Neither the United States Government nor the University of California nor any of their employees, makes any warranty, express or implied, or assumes any legal liability or responsibility for the accuracy, completeness, or usefulness of any information, apparatus, product, or process disclosed, or represents that its use would not infringe privately owned rights. Reference herein to any specific commercial product, process, or service by trade name, trademark, manufacturer, or otherwise, does not necessarily constitute or imply its endorsement, recommendation, or favoring by the United States Government or the University of California. The views and opinions of authors expressed herein do not necessarily state or reflect those of the United States Government or the University of California, and shall not be used for advertising or product endorsement purposes.

This report has been reproduced directly from the best available copy.

Available to DOE and DOE contractors from the

Office of Scientific and Technical Information

P.O. Box 62, Oak Ridge, TN 37831

Prices available from (615) 576-8401, FTS 626-8401

Available to the public from the

National Technical Information Service

U.S. Department of Commerce

5285 Port Royal Rd.

Springfield, VA 22161 


\title{
Observation of the Saturation of Langmuir Waves Driven by Ponderomotive Force in a Large Scale Plasma
}

R. K. Kirkwood, D. S. Montgomery1', B. B. Afeyan, J. D. Moody, B. J. MacGowan, S. H. Glenzer, W. L. Kruer, K. G. Estabrook, K. B. Wharton E. A. Williams, and R. L. Berger

University of California, Lawrence Livermore National Laboratory, P. O. Box 808, Livermore Ca. 94551

${ }^{1}$ Los Alamos National Laboratory, Los Alamos, New Mexico 875452

\begin{abstract}
We report the observation of amplification of a probe laser beam ( $\leq 1 \times 10^{14}$ $\left.\mathrm{W} / \mathrm{cm}^{2}\right)$ in a large scale $(\sim 1 \mathrm{~mm})$ plasma by interaction with a pumping laser beam $\left(\mathrm{I}=2 \times 10^{15} \mathrm{~W} / \mathrm{cm}^{2}\right)$ and a stimulated Langmuir wave. When the plasma density is adjusted to allow the Langmuir wave dispersion to match the difference frequency and wave number of the two beams, amplification factors as high as $\times 2.5$ result . Interpretation of this amplification as scattering of pump beam energy by the Langmuir wave that is produced by the ponderomotive force of the two beams, allows the dependence of Langmuir wave amplitude on ponderomotive force to be measured. It is found that the Langmuir wave amplitude saturates at a level that depends on ion wave damping, and is generally consistent with secondary ion wave instabilities limiting its growth.
\end{abstract}

PACS: $52.40 . \mathrm{Nk}, 42.65 . \mathrm{Hw}, 52.35 . \mathrm{Mw}$ 
The scattering of laser energy by large amplitude Langmuir waves has long been recognized as an important loss mechanism in laser driven inertial confinement fusion research [1]. The scattering waves are driven to large amplitude by the three wave process of stimulated Raman scattering (SRS). At moderate laser intensities the three wave process can be limited only by convective saturation resulting from the propagation of energy out of the interaction volume by one of the decay waves. When the convective saturation level is sufficiently high, as in large scale plasmas, Langmuir waves with amplitudes well below the convective saturation level, will decay by subsequent stimulation of ion acoustic, Langmuir, and electromagnetic waves. These secondary decay products can deplete the energy of the primary Langmuir wave sufficiently that it can not grow to the convective saturation limit but rather, will have its amplitude determined by the physics of the secondary process $[2,3,4,5,6]$. Early experiments have shown that Langmuir waves and ion waves anti-correlate in time suggesting that they are nonlinearly coupled $[7,8,9]$. More recent experiments have shown that, under conditions similar to what is expected in ignition experiments, the SRS reflectivity is dependent on the damping rate of the ion acoustic wave

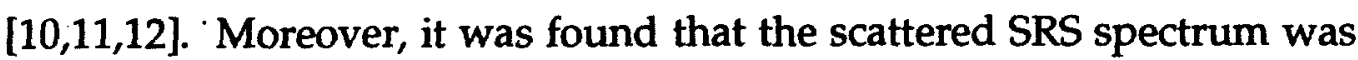
consistent with Langmuir wave amplitudes that tracked the thresholds for the secondary decay instabilities [10]. These observations suggest that the Langmuir wave response to the ponderomotive force is saturated by the secondary process when its amplitude is above the threshold for the process, similar to what was predicted by analysis [2] and observed in numerical calculations $[3,4,5]$. A separate line of experimentation using crossing laser beams [13] has shown that non-linearities in the response of plasma waves to 
ponderomotive force can be measured and that ion acoustic waves have a linear response up to large amplitude $\left(\sim 1 \% n / n_{c r}\right)$ in the high temperature $\mathrm{CH}$ plasmas studied.

In this Letter we report the first demonstration of the saturation of Langmuir waves driven by ponderomotive force under conditions relevant to ignition experiments. The Langmuir waves are driven by the beating of two intersecting laser beams of widely disparate frequency, and scatter a measurable amount of energy from the high frequency beam to the low frequency beam indicating their amplitude. The amplitude of the Langmuir wave is thus found to be roughly independent of the ponderomotive drive when the drive is large and when the damping rate of the ion acoustic wave is low, as predicted by models of secondary decays [2-6]. The experiments are done in low $Z$ plasmas which have electron temperatures [14], densities and scale length similar to what is expected in ignition scale experiments [15], and support the interpretation of the observed dependence of SRS reflectivity on the damping rate of the ion acoustic wave $[10,11,12]$ as due to saturation by secondary decay.

The experiments were performed at the ten beam Nova laser facility, using eight of the beams to pre-heat a gas filled target and the remaining two beams as interaction beams. The target consists of a $3000 \AA$ thick polyimide bag that is filled with atmospheric pressure gas so that it is nearly spherical with a radius of $1.3 \mathrm{~mm}$ when placed in the vacuum chamber [14]. The target is pre-heated with eight of the $f / 4.3$ beams that are defocused (converging) to have a diameter of $\sim 1.7 \mathrm{~mm}$. The heater beams have a $1 \mathrm{~ns}$ square pulse that produces a plasma that is hot and relatively homogeneous in the region inside a $1 \mathrm{~mm}$ radius and during latter half of the heating pulse ( 0.5 to $1.0 \mathrm{~ns}$ ). The target is filled with a $1 \mathrm{~atm}$. pressure mixture of the following gases: 
roughened fused silica. The plate is imaged onto a gated optical imager and onto the cathode of a photo diode that provides a measure of the transmitted probe power as a function of time [17]. The response time of the photo diode measurements is improved to better than 150 ps by deconvolving the impulse response of the system determined from measurements of a beam with a square incident pulse with no target present in the chamber.

Measurements of the transmission of the probe beam were performed over a range of plasma densities and compared with the transmission from an identical experiment with no probe beam. The initial density in each experiment was determined by the gas fill pressure and the molecular composition of the gas. To study plasma with strongly damped ion acoustic waves $\left(\operatorname{Im}[\omega] / \omega_{\mathrm{ia}} \sim 0.2\right)$ the targets are filled with mixtures of $\mathrm{CH}_{4} \mathrm{C}_{3} \mathrm{H}_{8}$, and $\mathrm{C}_{5} \mathrm{H}_{12}$ in various ratios, allowing the density to be varied from $5.6 \%$ to $8.2 \%$ of the critical density for $351 \mathrm{~nm}$ light. The transmitted probe power in the absence of a pump beam (pump off) was only weakly sensitive to the density in this range, while the transmitted power in the presence of the pump beam (pump on) was observed to have a substantial enhancement compared to the pump off case when the initial plasma density was in the vicinity of $n / n_{\mathrm{cr}}=$ $7.1 \%$. Figure 2 shows the time history of the transmitted probe power near the resonant value of density for both the pump on and pump off cases. The observed transmission in the pump off case is consistent with attenuation of the incident beam (196 GW) by inverse bremsstrahlung absorption when account is taken of scattered light and spreading of the transmitted beam [17]. In the pump on case the probe transmission is observed to be strongly enhanced during the 0.5 to $1.5 \mathrm{~ns}$ window that both pump and probe are on, indicating significant energy is transferred from the pump beam. The amplification is measured during the 0.5 to $1.0 \mathrm{~ns}$ time period by averaging 
the transmitted power over this time period and taking the ratio of the average power in the pump on case to that in the pump off case. This amplification is $2.15( \pm .3)$ for the case shown in figure 2 . The interaction is found to be strongly resonant with plasma density as observed in the plot of amplification vs. target initial electron density (fill pressure) shown in figure 3. The data in figure 3 is restricted to cases of low probe beam intensity and strong ion acoustic wave damping, to avoid any Langmuir wave nonlinearity which would result in a reduction of the measured amplification. The maximum of amplification observed at $7.1 \%$ of the critical density combined with the observation of little or no affect of the pump (amplification 1) that is observed at both higher and lower density in figure 3 , are consistent with the calculated Langmuir wave resonance for a plasma with the measured electron temperature (2.1 keV) [14], and support the interpretation that the amplification is due to a resonant three wave interaction of the two beams and a Langmuir wave in the plasma.

Having identified a density that is sufficiently near the Langmuir wave resonance in this plasma $\left(n / n_{c}=7.1 \%\right)$ and determined the magnitude of the amplification when the Langmuir wave amplitude is small, experiments were performed to determine the non-linear response of Langmuir waves at larger amplitude by both increasing the probe beam intensity and reducing the damping of the ion acoustic wave by reducing the fraction of protons in the plasma. These experiments show that the amplification is substantially reduced both by increasing the probe intensity or by reducing the damping of the ion acoustic wave while holding the plasma density close to the resonant value (that is within the shaded region in figure 3). For example the amplification observed at low probe power $(65 \mathrm{GW})$ under the conditions of figure 2 is 2.5 while the amplification in an identical experiment with a probe 
power of $500 \mathrm{GW}$ is less than 1.32 (where 1.0 represents no resonance). Similarly the amplification is reduced to less that 1.15 by changing the gas from $99 \% \mathrm{C}_{3} \mathrm{H}_{8}\left(+1 \% \mathrm{Ar}\right.$ ) to $79 \% \mathrm{CO}_{2}+20 \% \mathrm{CF}_{4}$ (+ $1 \% \mathrm{Ar}$ ) which maintains the electron density but reduces the ion acoustic wave damping by a factor of $\sim 20$ [16]. These observations are used to show the saturation of the Langmuir wave by interpreting the amplification of the probe beam as a measurement the energy the Langmuir wave scatters from the pump beam which is interpreted as the Langmuir wave amplitude. This analysis can be understood by considering the total power scattered from the pump beam rather than the amplification of the probe. The power scattered from the pump is determined as the difference between the transmitted probe power in the case with the pump on and with the pump off, again averaging over the 0.5 to $1.0 \mathrm{~ns}$ period when all beams are on. This scattered power is corrected for the inverse bremsstrahlung between the point where the center of the beams cross and the outer edge of the plasma, and plotted vs. the incident probe power and ion acoustic wave damping for constant pump power in figure 4. Because the power scattered by the Langmuir wave is proportional to the square of its amplitude $(\delta n / n)^{2}$ and the probe beam intensity is proportional to the square of the ponderomotive force driving the Langmuir wave, a linear Langmuir wave response would be represented by data that parallels the linear scaling curve shown in figure 4. Clearly for all the damping rates studied the scattered power falls below the linear scaling when the probe power is greater than $200 \mathrm{GW}$ demonstrating that the Langmuir wave amplitude is non-linearly saturated at high ponderomotive drive. Moreover, the saturated (or maximum) level of scattering is observed to depend on the ion acoustic wave damping rate in the experiments with different materials. It is shown in figure 4, that high ion wave damping leads 
to a high level of scattering and low ion wave damping leading to unmeasurable scattering, as is consistent with the model of Langmuir wave amplitude being saturated by a secondary decay of the Langmuir wave into an ion acoustic wave and a third wave [2-6,10-12]. This non-linearly saturated response of the Langmuir wave to the ponderomotive force produced by the beating of the two laser beams, and its dependence on the damping rate of the ion acoustic wave, is the primary result of this work.

Further the comparison of the observations with theories of secondary decay of the Langmuir wave [2-6] can be made by estimating the amplitude of the wave from the fraction of power it scatters from the pump beam. Such an estimate is made using a 1-D model $[10,18]$ that neglects inhomogeneities in the incident and scattered beams and gives a lower limit to the scattering wave amplitude. This model gives the relationship between the fraction of pump power scattered $(F)$ and the wave amplitude $(\delta n / n)$ in terms of the normalized plasma density $\left(n / n_{c}\right.$ where $n_{c}$ is the critical density of the incident $351 \mathrm{~nm}$ beam), the incident beam wave number, and two characteristic lengths; the system size (L), and the correlation length of the Langmuir waves $(\Delta k)^{-1}$.

$$
\mathrm{F} \leq \frac{1}{4}\left(\frac{\mathrm{n}}{\mathrm{n}_{\mathrm{co}}}\right)^{2} \mathrm{k}_{0}{ }^{2} \mathrm{~L}(\Delta \mathrm{k})^{-1}\left(\frac{\delta \mathrm{n}}{\mathrm{n}}\right)^{2}
$$

This formula can also be derived from a 3-D analysis in which the Langmuir wave are assumed to have a phase velocity much less than the speed of light, the wave amplitude is small, the incident and scattered waves are nearly parallel, and the Langmuir waves are incoherent in at least one dimension characterized by $(\Delta k)^{-1}[19]$. The coherence length of the Langmuir waves is determined by the width of their $\mathrm{k}$ spectrum and is difficult to measure. If the plasma response is linear the $\mathrm{k}$ spectrum of the waves will be a 
convolution of the $\mathrm{k}$ spectrum of the driving ponderomotive force and the plasma response function. The width of the $\mathrm{k}$ spectrum of the driving ponderomotive force is then a lower limit to the width of the spectrum of the Langmuir waves which would be approached in a homogeneous plasma. Moreover a non-linear plasma response will usually only further broaden the Langmuir wave spectrum. As a result, we use width of the $k$ spectrum of the incident beams as an upper limit to the correlation length of the Langmuir waves. Since the ponderomotive force is produced by the beating of two $\mathrm{f} / 4.3$ beams intersecting at $25^{\circ}$ from anti-parallel, it can be coherent over a distance no greater that the speckle length of these two beams; $(\Delta \mathrm{k})^{-1} \leq$ $1.2 \times 10^{6} \mathrm{~m}^{-1}$. Using this length and the experimental plasma parameters in Eq. 1 the amplitude of the scattering Langmuir wave is estimated from the measurements of fraction of pump beam power that the wave scatters, as shown on the right axis on in Fig. 4. This fraction is calculated using the pump beam power that is determined by the incident power and a correction for inverse Bremsstrahlung between the plasma edge and the point at which the center of the two beams cross. The results are compared with the threshold for the secondary decay instability shown as dashed lines in figure 4. The threshold is determined from the damping rates of both of the secondary decay products and can be written in terms of the Langmuir wave amplitude as

$$
\left(\frac{\delta \mathrm{n}}{\mathrm{n}}\right)_{\mathrm{LDI}}=4 \mathrm{k}_{\mathrm{L}} \lambda_{\mathrm{D}}\left(\frac{v_{\mathrm{ia}}}{\omega_{\mathrm{ia}}}\right)^{1 / 2}\left(\frac{v_{3}}{\omega_{3}}\right)^{1 / 2}
$$

where $k_{L}$ is the wave number of the primary Langmuir wave that is driven by the beating of the two beams, $\lambda_{D}$ is the Debye length, $\omega_{i a}$ and $v_{i a}$ are the real and imaginary parts of the frequency of the ion acoustic decay product, and $\omega_{3}$ and $v_{3}$ represent the frequency of the third wave. When the third wave is an 
electromagnetic wave, the instability is the Electromagnetic Decay Instability (EDI) and $\omega_{3}$ and $v_{3}$ are approximately the plasma frequency and the collisional damping rate. When the third wave is a Langmuir wave, the instability is the Langmuir Decay Instability (LDI) and $\omega_{3}$ and $v_{3}$ are approximately the plasma frequency and the electron landau damping rate. Recent analysis of the non-Maxwellian distribution of electrons that may be produced in these plasmas by mechanisms such as collisional absorption of the beams, non-linear Landau damping of the Langmuir waves, or non-local heat transport [20], has shown that the electron Landau damping rate may be much lower than in a Maxwellian plasma and the damping rate of the Langmuir wave may approach the collisional damping rate as a lower limit. As a result a sufficient condition for the presence of EDI or LDI is that the $\delta \mathrm{n} / \mathrm{n}$ estimated from the measured scattered power (points in figure 4) is greater than or equal to the threshold given by Eq. 2 evaluated with $\omega_{3}$ and $v_{3}$ equal to the plasma frequency and the collisional damping rate (dashed lines in figure 4). This threshold is found to correspond closely with the data as shown, supporting the interpretation that the observed Langmuir wave saturation is due to a secondary decay process involving ion waves.

\section{References}

[1] See for example W. L. Kruer 'The Physics of Laser Plasma Interactions' (Addison-Wesley Publishing Co. Redwood City, CA, 1988).

[2] S. J. Karttunen, Plasma Physics 22, 151 (1980).

[3] G. Bonnaud, Denis Pesme, and Rene Pellat, Phys. Fluids B 2, 1618 (1990).

[4] B. Bezzerides, D. F. Dubois, and H. A. Rose, Phys. Rev. Lett. 70, 2569 (1993).

[5] T. Kolber, W. Rozmus, and V. T. Tikhonchuk, Phys. Fluids B 5, 138 (1993).

[6] K. L. Baker, Ph.D. Dissertation, University of California, Davis, 1996; see also P. K. Shukla et al., Phys Rev. A 27, 552 (1983).

[7] C. J. Walsh, D. M. Villeneuve, and H. A. Baldis, Phys. Rev. Lett. 53, 1445 (1984).

[8] H. A. Baldis et al., Phys. Rev. Lett. 62, 2829 (1989).

[9] D. S. Montgomery et al., Submitted to Phys. Rev. Lett.

[10] R. K. Kirkwood et al., Phys. Rev. Lett. 77, 2706 (1996). 
[11] R. K. Kirkwood et al., to appear in Physics of Plasmas 4, Vol. 5 (1997).

[12] J. C. Fernandez et al., Phys. Rev. Lett. 77, 2702 (1996).

[13] R. K. Kirkwood et al., Phys. Rev. Lett 77, 2065 (1996).

[14] S. H. Glenzer et al., Phys. Rev. E 55, 927 (1997).

[15] B. J. MacGowan et al., Physics of Plasmas 3, 2029 (1996).

[16] E. A. Williams et al., Physics of Plasmas 2, 129 (1995).

[17] J. D. Moody et al., to appear in Rev. Sci. Inst.

[18] T. Kolber, W. Rozmus, and V. T. Tikhonchuk, Phys. Plasmas 2, 256 (1995).

[19] R. K. Kirkwood et. al. in preparation.

[20] B. B. Afeyan, et al., submitted to Phy. Rev. Lett. 


\section{Figure Captions}

Figure 1 Geometry of the crossing beam experiment showing a short wavelength, high intensity pump beam and a long wavelength low intensity probe beam intersecting in a spherical low density plasma. The transmitted beam diagnostic measures the time history of the transmitted power of the probe beam.

Figure 2 Power transmitted through the plasma by the probe beam for the cases with and with out a pump beam. The transmission of the probe beam is enhanced by a factor of $\geq 2$ during the time period that the pump is on, indicating that a large amplitude Langmuir wave is being stimulated and scattering substantial energy.

Figure 3 The amplification measured during the 0.5 to $1.0 \mathrm{~ns}$ time period is shown as a function of plasma density for the case of low probe intensity and strong ion acoustic wave damping (ie; unsaturated Langmuir waves). A maximum is observed at $\mathrm{n} / \mathrm{n}_{\mathrm{c}}=7.1 \pm 0.15 \%$ consistent with the Langmuir resonance.

Figure 4 The additional power scattered into the probe beam when the pump beam is present (scattered power) and the density is resonant is plotted vs. the incident power in the probe for three different gas fills that have different damping rates for the ion acoustic wave $\left(v_{\mathrm{ia}} / \omega_{\mathrm{ia}}\right)$. The solid line represents a linear relationship between the amplitude of the scattering Langmuir wave and the probe power (ponderomotive force), while the data show a weaker dependence on probe power when the wave amplitude is high (ie; saturation). The dashed lines represent the threshold of the secondary decay instability as discussed in the text. 


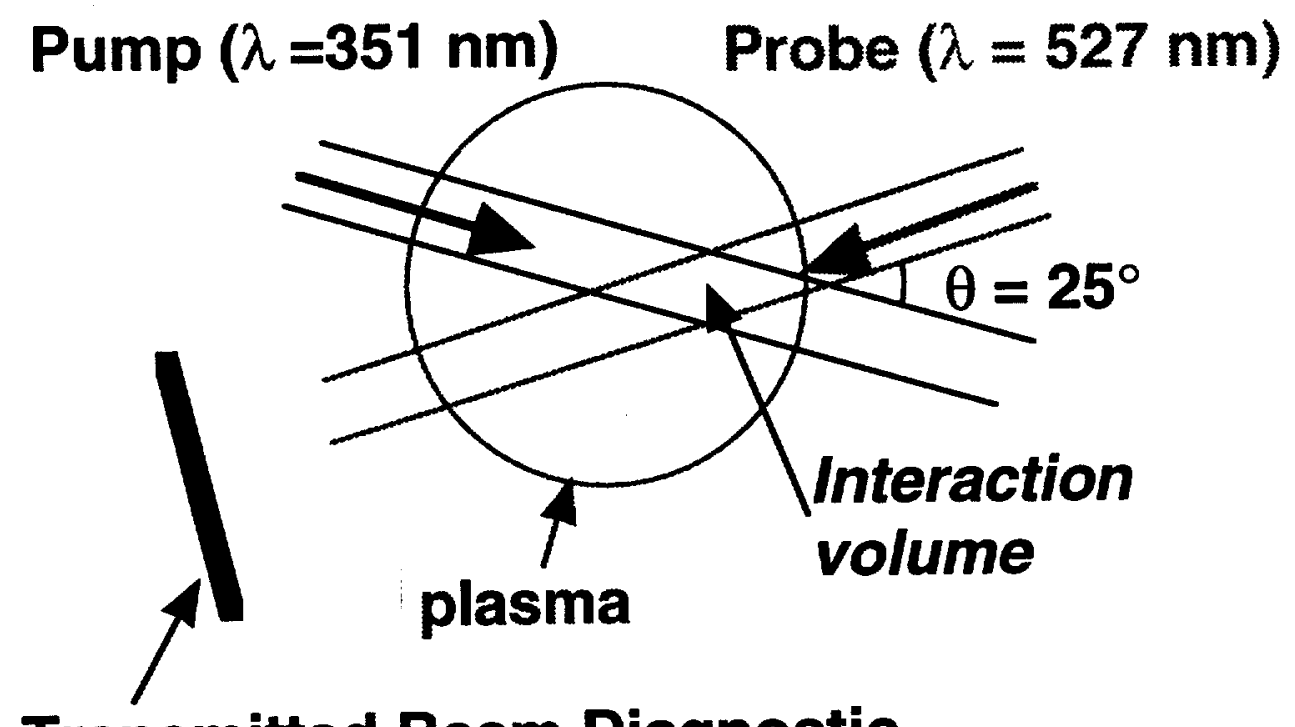

Transmitted Beam Diagnostic

Figure 1

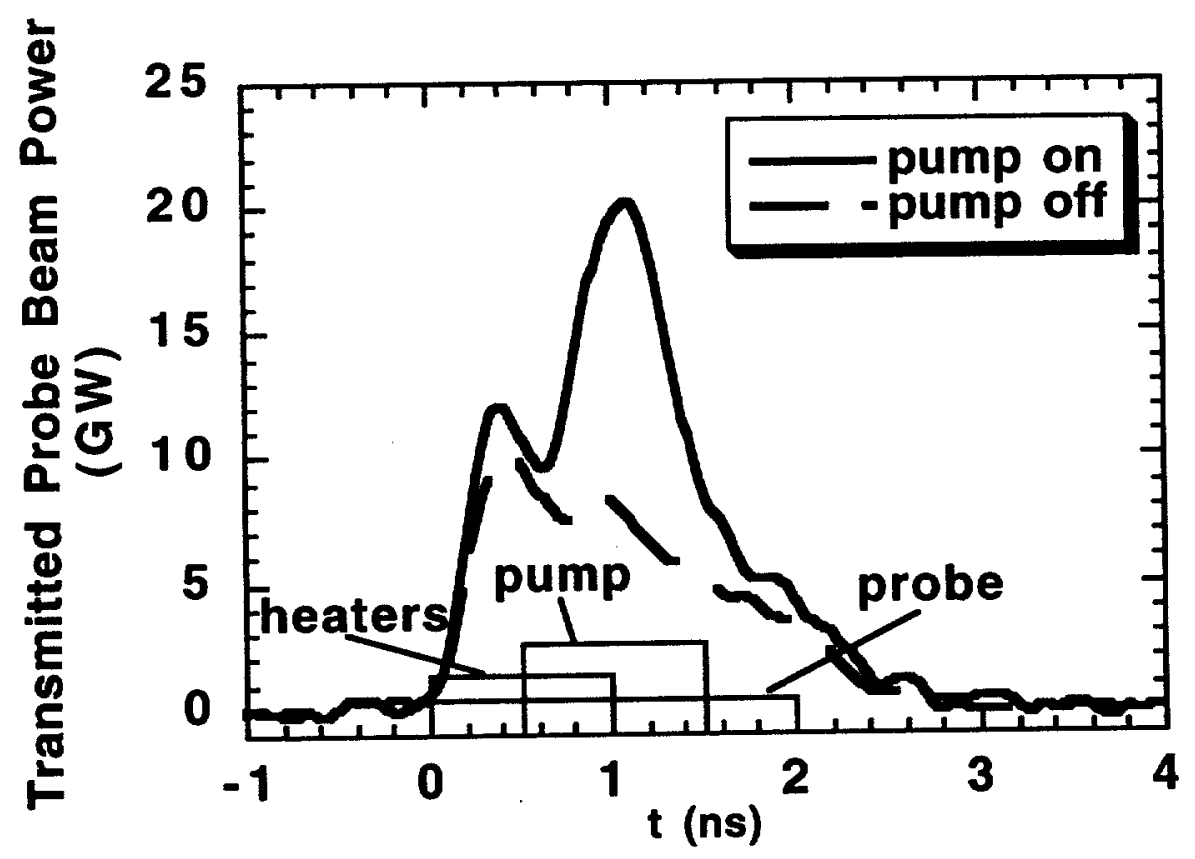

Figure 2 


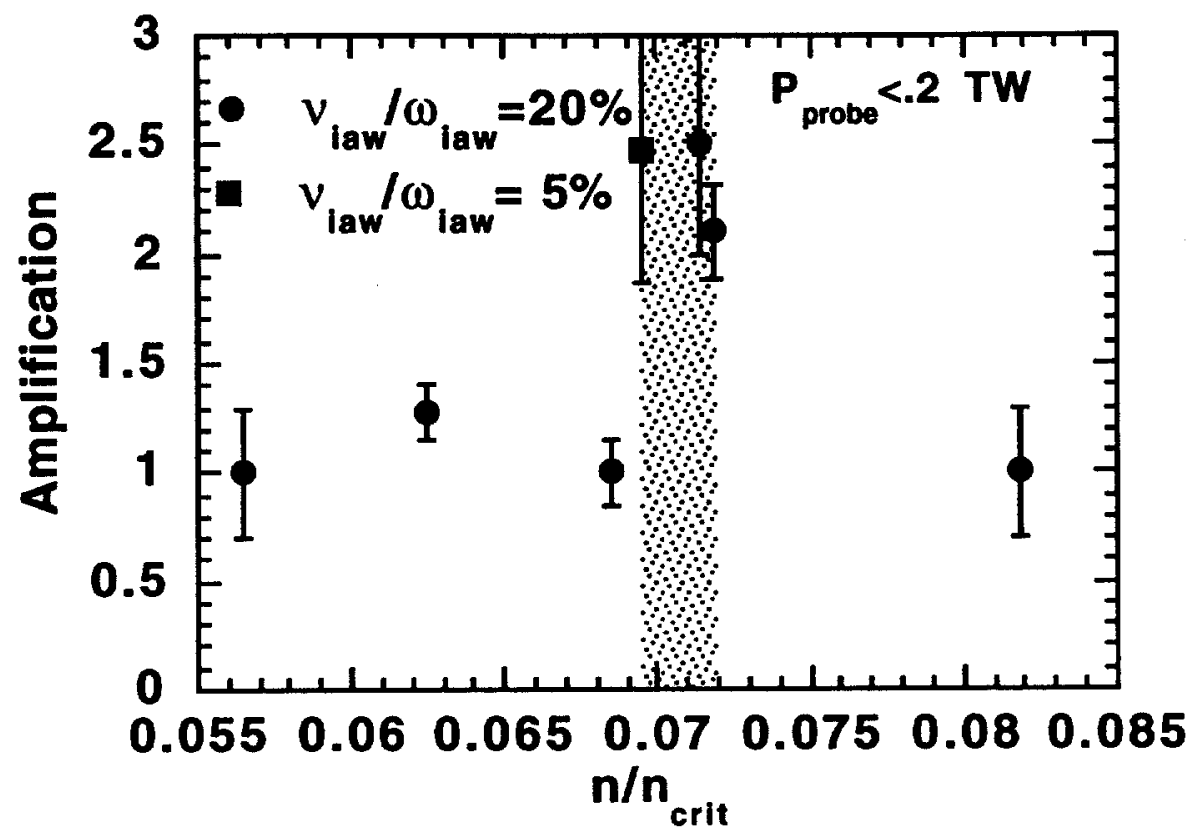

Figure 3

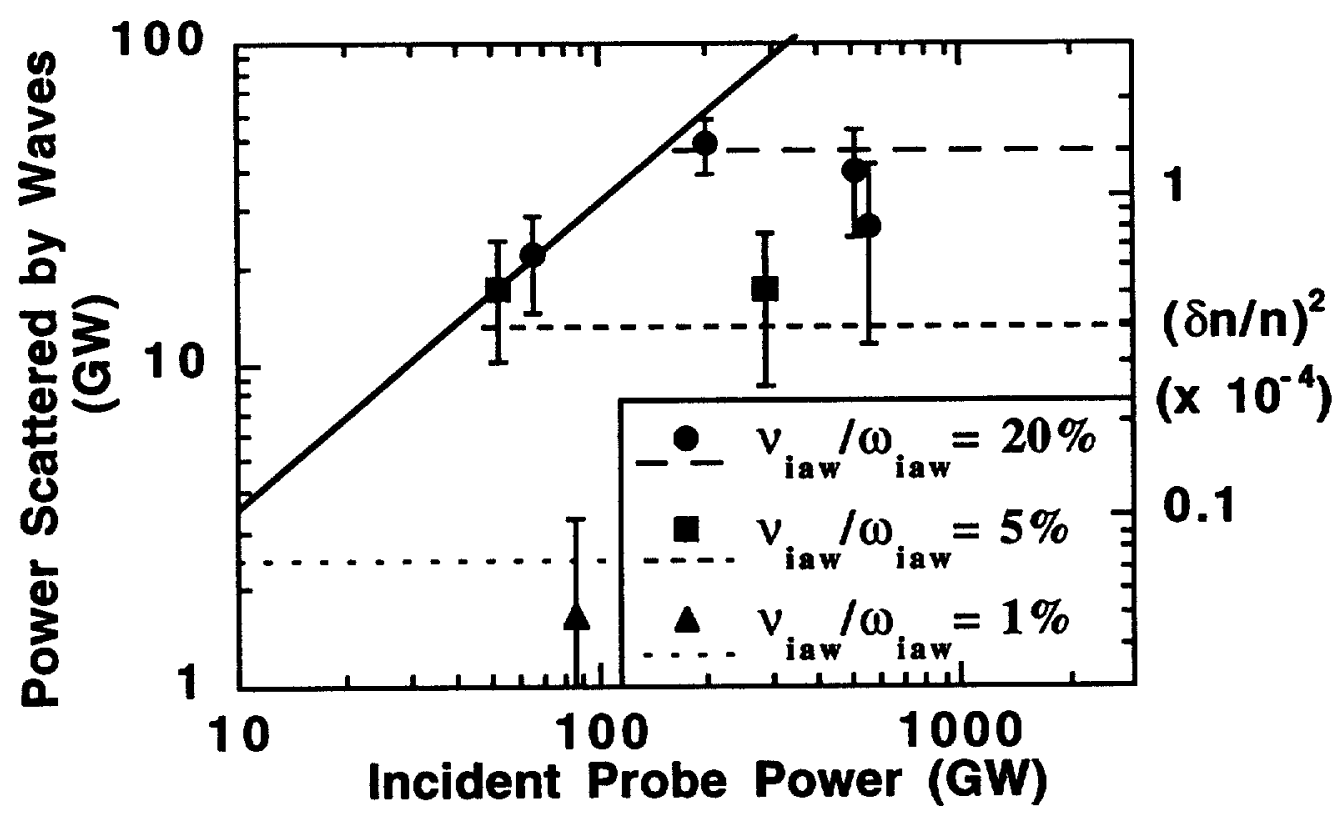

Figure 4 


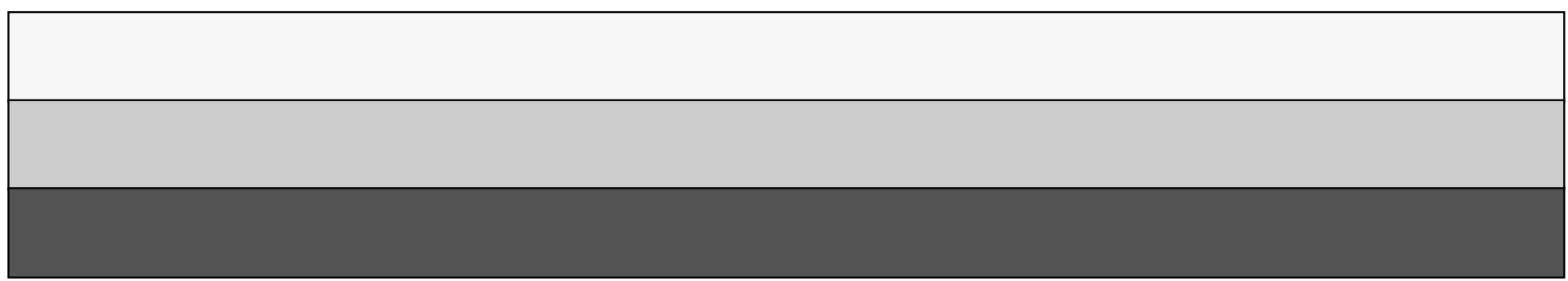

